\title{
Telemedicine visits in an established multidisciplinary central nervous system clinic for radiation oncology and neurosurgery (RADIANS) in a community hospital setting
}

\author{
Bowen SC ${ }^{1,2}$, Gheewala R ${ }^{3}$, Paez WA ${ }^{4,5}$, Lucke-Wold B ${ }^{6}$, Mitin $\mathrm{T}^{4,5}$, Ciporen $\mathrm{JN}^{1,2}$ \\ Department of Neurological Surgery, Oregon Health \& Science University, Portland, U.S.A. \\ Brandon.Lucke-Wold@neurosurgery.ufl.edu
}

\begin{abstract}
OBJECTIVE: To determine the impact of telemedicine visits, compared to in-person visits, on patient satisfaction in an established community hospital-based multidisciplinary central nervous system (CNS) clinic. METHODS: Telemedicine options - virtual visits and teleconferencing - were introduced in July 2020. Both radiation oncologist and neurosurgeon were simultaneously present for the telemedicine visit. Descriptive patient demographics, survey responses, and travel time and distance calculations were analyzed. Satisfaction score was compared to previously published data.

RESULTS: A total of twenty-five telemedicine visits ( $n=22$ video; $n=3$ phone) were completed since July 2020. Patient demographics are as follows: mean age was 59 years (range $=22-81$ ), women (9) and men (16), repeat telemedicine visits $n=10$, malignant CNS disease (17) and benign disease (5). Mean oneway distance traveled was 165.07 miles (median=114; range $=0.8-358$ ). Mean roundtrip travel time was estimated at $5 \mathrm{~h} 5 \mathrm{~min}$. Mean telemedicine visit duration was 15.3 mins (range=4-46). Mean patient satisfaction score for telemedicine visits was 4.84 .

CONCLUSION: Patients who opted for the telemedicine visits found them just as effective as in-person visits, saving time and travel costs as well as ensuring patient safety during the current COVID-19 pandemic. The telemedicine visit platform facilitates the multidisciplinary clinic model and should be considered for more widespread utilization (Tab. 3, Fig. 1, Ref. 18). Text in PDF www.elis.sk. KEY WORDS: central nervous system, community hospital, multidisciplinary, neurosurgery, radiation oncology, telemedicine data, RADIANS.
\end{abstract}

\section{Introduction}

With the first reported COVID-19 cases reaching the United States in January 2020, the World Health Organization (WHO) declared the outbreak a global pandemic in March 2020 (1, 2). Increasing US prevalence forced the Centers for Disease Control and Prevention (CDC) to implement public safety measures to minimize exposure in the form of early diagnosis, contact tracing, mandated stay-at-home orders and mask wearing (3). While guidelines were in place to promote reduced COVID-19 transmission, it created challenges for patients to continue routine care (1, 3). Moreover, the resulting impact the pandemic had on cancer care was evidently seen in delays in cancer screening, diagnosis

${ }^{1}$ Department of Neurological Surgery, Oregon Health\&Science University, Portland, OR, U.S.A., ${ }^{2}$ Department of Neurological Surgery, OHSUHillsboro Medical Center, Hillsboro, OR, U.S.A., ${ }^{3}$ School of Medicine, Oregon Health\&Science University, Portland, OR, U.S.A., ${ }^{4}$ Department of Radiation Medicine, Oregon Health\&Science University, Portland, OR, U.S.A., ${ }^{5}$ Department of Radiation Medicine, OHSU-Hillsboro Medical Center, Hillsboro, OR, U.S.A., and ${ }^{6}$ Department of Neurosurgery, University of Florida, Gainesville, FL, U.S.A.

Address for correspondence: B. Lucke-Wold MD, PhD, MCTS, Department of Neurosurgery, University of Florida, 1505 SW Archer Rd, Gainesville, FL 32608 U.S.A. and treatment, and declines in in-patient and in-office surgical procedures and overall patient encounters (4-9), particularly in the elderly age population (10). Given the disruption and risk of COVID-19 spread from in-person visits, healthcare delivery modifications needed to be employed to fit these restrictions. Given the increased access to technology and the internet, the use of telemedicine, which is the remote delivery of healthcare via secure video conferencing or telephone conferencing, was more widespread and became a viable solution to this dilemma $(11,12)$. Important to note, telemedicine has been previously explored as a potential means of disease control and clinical care during times of epidemics, most recently during the Ebola virus outbreak (13).

The RADIation oncology And NeuroSurgery (RADIANS) multidisciplinary clinic was formed at a community hospital in 2016 as a collaboration between radiation oncology and neurosurgery physicians for simultaneous evaluation of central nervous system (CNS) disease (14). The clinic is unique both in its multidisciplinary model and community hospital setting. Patients referred to the RADIANS clinic are seen and evaluated in one visit by both a radiation oncologist and neurosurgeon. Patients are then followed long-term to detect new metastasis or disease recurrence. Many RADIANS patients have cancer, multiple comorbidities and a higher risk of severe illness or mortality from COVID-19, thus, 
Tab. 1. Demographics of patients seen via telemedicine in RADIANS multidisciplinary clinic between July 2020 and February 2021, n= 25 appointments.

\begin{tabular}{lc}
\hline Age (years) & 59 \\
Mean & 65 \\
Median & $22-81$ \\
Range & \\
\hline Sex n, (\%) & $9(36)$ \\
$\quad$ Female & $16(64)$ \\
$\quad$ Male & \\
\hline One-way distance from RADIANS clinic (miles) & 161.5 \\
$\quad$ Mean & 114 \\
Median & $0.8-358$ \\
Range & \\
Visit Type n, (\%) & $20(80)$ \\
Video only & $1(4)$ \\
Telephone only & $2(8)$ \\
Video converted to telephone & $1(4)$ \\
Video and telephone & \\
\hline Number of repeat telemedicine visits & 15 \\
First time telemedicine appointment & 10 \\
Repeat telemedicine appointment & \\
Consultation Time (minutes) & 15.3 \\
$\quad$ Mean & 10 \\
Median & $4-46$ \\
Range
\end{tabular}

Tab. 2. Metastatic cancer types and central nervous system lesions for patients evaluated via telemedicine in RADIANS clinic between July 2020 and February $2021 \mathrm{n}=25$ appointments.

\begin{tabular}{|c|c|}
\hline Malignant & \\
\hline Primary brain & \\
\hline Glioblastoma & 4 \\
\hline Oligodendroglioma & 2 \\
\hline Metastatic Brain & \\
\hline NSCLC* & 4 \\
\hline SCLC* & 1 \\
\hline Ovarian & 2 \\
\hline Primary Spine & 0 \\
\hline Metastatic Spine & \\
\hline Prostate & 1 \\
\hline Metastatic Spine and B & \\
\hline NSCLC** & 3 \\
\hline Breast & 1 \\
\hline Benign & \\
\hline Brain & \\
\hline Meningioma & 2 \\
\hline Schwannoma & 1 \\
\hline Spine & \\
\hline Osteoblastoma & 1 \\
\hline No active disease/unkn & \\
\hline Brain & \\
\hline NSCLC* & 2 \\
\hline Spine & \\
\hline NSCLC* & 1 \\
\hline
\end{tabular}

prevention of COVID-19 exposure is crucial (4). To increase patient and provider protection, the RADIANS clinic began offering telemedicine follow-up visits as an option.

\section{Materials and methods}

From July 2020 to February 2021, patients in the RADIANS clinic were offered appointments via telemedicine. Patient clinical and demographic data were collected and stored in a secure database for access to IRB-approved research members. Patients who opted for a telemedicine visit, were evaluated either through secure video conferencing or telephone. Both radiation oncologist and neurosurgeon were simultaneously present for the entire visit, typically in the same room, sharing one video screen for the appointment, although one appointment was conducted with the providers joining the video visit from their respective offices. With the consent of the patient, family members and/or caregivers were also part of the telemedicine appointment. After the appointment patients were called by the research assistant and asked to verbally provide answers for two qualitative surveys. Informed consent was obtained from the participants. One survey was a follow-up patient satisfaction survey about the multidisciplinary CNS clinic model (follow-up survey) and is given to all patients. The other survey was a patient satisfaction survey about the telemedicine appointment (telemedicine survey) which was adapted from the survey used by Fieux et al (16). Survey responses, travel distance, and travel time were analyzed. Travel time was calculated using a speed of $65 \mathrm{mph}$ and mean round trip mileage. Mean general survey patient satisfaction score was compared to previously published patient satisfaction score from in person appointments (17).

\section{Results}

There were 26 telemedicine appointments conducted from July 6th, 2020 to February 1st, 2021. Of these 26 visits, 25 completed the after-visit surveys. There were 22 virtual appointments (with or without telephone conferencing for sound technical difficulties) and 3 telephone appointments (Tab. 1). A total of 15 patients were surveyed, 10 of the appointments were previously seen patients who had 2 or more appointments via telemedicine (Tab. 1). Due to patients receiving the survey after each appointment, the results are based upon $n=25$ appointments rather than $n=15$ patients. The appointments were typically to follow-up after completion of treatment or to review new imaging. Patient demographics are presented in Table 1 . The mean patient age was 59 years, with 9 women and 16 men. Had the appointment been in the clinic, patients would have travelled an average of 165.07 miles one way (median = 114 miles; range $=0.8-358$ miles). Mean round trip travel time was estimated to be 5 hours and 5 minutes. The length of the telemedicine visit was 4 minutes to 46 minutes, the average length being 15.3 minutes.

In 17 appointments the patient had a malignant CNS disease, in 5 of the visits the patient had a benign CNS disease, and in 3 of the visits the patient was found to not have a CNS disease or had a CNS disease with unknown etiology (Tab. 2).

Figure 1 shows the mean response to question 1 of the followup survey, which asked patients to "Please rate (their) overall satisfaction with being followed up at this clinic" graded on the 1-5 Likert scale where $1=$ not satisfied and 5 = very satisfied. Mean patient satisfaction score for telemedicine visits was 4.84 . This 


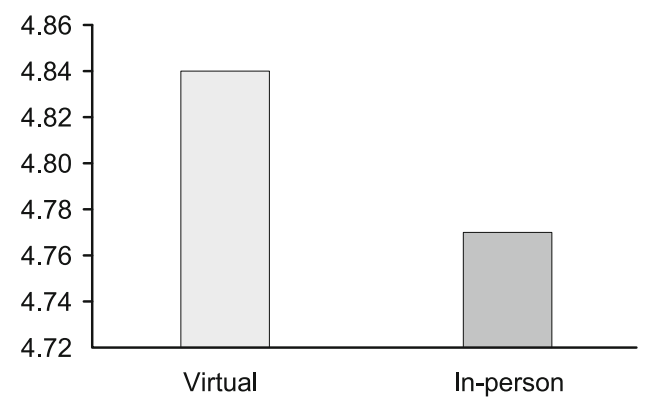

Fig. 1. Responses to follow-up survey question 1.) "Please rate your overall satisfaction with being followed up at this clinic" with 1-5 Likert scores by patients seen via telemedicine and in person. Results are reported as a mean of responses (telemedicine $n=25$, in-person $n=101$ ). The Likert scale was scored as: $1=$ not satisfied, $5=$ very satisfied.

patient satisfaction score was compared to the patient satisfaction score of 4.79 for visits in the clinic (16).

Table 3 shows the average qualitative results to the telemedicine survey. Patient were satisfied with the video quality, found it easy to communicate to the physicians, appreciated screenshare to review new imaging, felt telemedicine was just as effective as an in-person appointment, saved money/time by meeting via telemedicine, and would use telemedicine again for future appointments. They also denied feeling nervous communicating via telemedicine, confusion talking to two providers via telemedicine, and feeling irritated by the lack of a physical exam.

\section{Discussion}

The COVID-19 pandemic helped our clinic to offer telemedicine visits, virtual visits or teleconsultations. With pre-established patient satisfaction levels for the in-person RADIANS clinic, there can be a comparison of the satisfaction of patients seen via telemedicine to those seen in-person. The study also sought to evaluate the patient's experiences with multidisciplinary telemedicine, as well as time and cost burdens that telemedicine visits potentially alleviate.

Telemedicine visits enable simultaneous multidisciplinary CNS evaluation with both radiation oncologist and neurosurgeon, particularly during the COVID-19 pandemic. The RADIANS multidisciplinary clinic model demonstrated a continued high patient satisfaction rating both for telemedicine appointments and inperson appointments (17) indicating a high level of consistency in the quality of care provided in both visit types.

Patients were overall very satisfied with the telemedicine consults and would elect to have future telemedicine appointments (some patients have had multiple telemedicine visits). While 23 of the visits were scheduled to be virtual visits, technical difficulties required some modifications. One appointment was converted to a teleconsultation due to the patient losing internet access and two appointments used a combination of a virtual and telephone appointment due to the sound not functioning for the patient and/ or the provider. By meeting virtually, even without sound on the video call, the providers were able to show the patient their images, something that could not be done if the appointment was just
Tab. 3. Telemedicine survey questions regarding telemedicine experience and patient response (mean) using 1-5 Likert scoring.

\begin{tabular}{|c|c|c|}
\hline Question & Results & $\begin{array}{l}\text { Standard } \\
\text { Deviation }\end{array}$ \\
\hline $\begin{array}{l}\text { 1. I was satisfied with the sound quality during } \\
\text { the appointment }\end{array}$ & 4.67 & 1.13 \\
\hline $\begin{array}{l}\text { 2. I was satisfied with the video quality during the } \\
\text { appointment * }\end{array}$ & 4.95 & 0.21 \\
\hline 3. It was easy to join the virtual consult* & 4.57 & 0.66 \\
\hline $\begin{array}{l}\text { 4. Communicating via telephone/video made me } \\
\text { nervous }\end{array}$ & 1.08 & 0.40 \\
\hline $\begin{array}{l}\text { 5. I could easily communicate and tell my doctor } \\
\text { my health problems }\end{array}$ & 4.92 & 0.28 \\
\hline $\begin{array}{l}\text { 6. It was confusing talking to two doctors at once } \\
\text { via video/telephone }\end{array}$ & 1.08 & 0.40 \\
\hline 7. I felt the doctors answered all my questions & 5.00 & 0.00 \\
\hline $\begin{array}{l}\text { 8. I appreciated that my providers could show me } \\
\text { my imaging via screenshare }^{\dagger}\end{array}$ & 5.00 & 0.00 \\
\hline $\begin{array}{l}\text { 9. I was satisfied with the doctor's response to my } \\
\text { problem }\end{array}$ & 4.92 & 0.28 \\
\hline $\begin{array}{l}\text { 10. I think the multi-disciplinary telemedicine ap- } \\
\text { pointment was as effective as visits in the clinic }\end{array}$ & 4.76 & 0.52 \\
\hline $\begin{array}{l}\text { 11. I was bothered that the doctors could not ex- } \\
\text { amine me }\end{array}$ & 1.12 & 0.44 \\
\hline 12. Telemedicine saved me travel time and/or money & 4.84 & 0.47 \\
\hline 13. I would use telemedicine again & 4.92 & 0.40 \\
\hline $\begin{array}{l}\text { 14. Overall, I was satisfied with the telemedicine } \\
\text { appointment }\end{array}$ & 4.96 & 0.20 \\
\hline
\end{tabular}

a teleconsultation. There are no significant prediction factors for patients that will struggle to use the virtual visit platform. Although a little over half of the appointments were with patients 65 years and older, who are generally thought to struggle with technology, patients still agreed that it was easy to join their virtual visit (Table 3 , question 3). Training on virtual visits for front desk staff was also crucial in ease of joining the visit. By assessing the technical difficulties encountered and barriers patients encounter when joining virtual visits, we could further prepare staff to assist patients with commonly encountered problems.

Though patients could not undergo a thorough and complete neurological exam via video, providers were nevertheless able to assess surgical incision healing, cranial nerve functions, gait, and gross motor function. When asked survey question 11 ("I was bothered that the doctor could not examine me"), some patients explained they were not dissatisfied because felt they were examined during their virtual visit. While some physical exam assessments need to be conducted in person, other parts of the examination can be modified to be conducted virtually. These exams were not possible for patients seen only by telephone. There have been published guidelines for modifying musculoskeletal and neurological examination techniques to be used effectively in virtual visits $(11,18)$.

Another unique and valuable feature of virtual visits, is the ability for the provider to share their screen, allowing the patients to view their imaging. Due to limited access to computers in the exam rooms, patients do not often review their images in-person. 
However, with virtual visits patient can view their images and further understand their disease with the providers guidance. All patients that viewed their imaging via screen share reported they were satisfied with this feature (Table 3, question 8). Given both the ability to conduct exams and share imaging, virtual visits are preferred over telephone visits in the telemedicine care model.

Given the unique specialization of the RADIANS clinic, patient's residences were in Oregon, Washington, and California - the furthest patient located 358 miles from the clinic. Telemedicine allows the clinic to see patients that otherwise may not have been able to easily access care, due to their distance from the clinic and/or living in a rural area without access to specialized healthcare providers. A telemedicine visit that may take as little as 4 minutes would likely not be worth the 716-mile round trip journey to Hillsboro, Oregon. Patients not only saved travel time and driving expenses, but also saved the likely expenses for accommodations, food, and time taken off for work. Fieux et al.(16) reported virtual ENT clinic estimated total savings of $\$ 48,000$ for 354 patients, in just travel costs. We estimate that an in-person RADIANS visit takes 45 minutes to complete. Consequently, telemedicine saved provider and clinic time, increasing opportunities to evaluate additional patients.

While both neurosurgeon and radiation oncologist are present in the RADIANS clinic, virtual visits could allow the providers to see a patient from their respective offices. One virtual visit was conducted with the providers joining the visit from their own office, rather than together. Virtually visits facilitate the multidisciplinary clinic model, with clinicians being able to collaborate without needing to be in the same room. While in-person appointments are still crucial, telemedicine is a valuable tool for creating multidisciplinary clinics and should continue to be offered as well as be considered for more widespread utilization, especially at large urban hospitals.

Although a limitation of the study is our sample size, our early experience is promising. Currently, telemedicine appointments are being offered and patient surveys administered. As more data is collected, further studies will be done to explore how telemedicine impacts caregiver burden and how it affects healthcare disparities in terms of access to care and telemedicine reliability. Patients that struggle with the technical aspects of virtual visits will continue to be tracked to identify common problems.

\section{Conclusion}

Patient appointments conducted via telemedicine were found to be just as effective as an in-person visit, saving time and travel costs, while ensuring patient safety during the current COVID-19 pandemic. Given that some RADIANS patients reside hundreds of miles away, offering telemedicine visits removes geographical barriers, thus improving access to care. Patient satisfaction with the RADIANS multidisciplinary clinic remained consistent across both telemedicine and in-person consultations. Most patients agreed they would likely use the telemedicine option for future followup. Our early findings strongly support offering telemedicine visits to patients. Additionally, providing a telemedicine option for patients should be standardized in multidisciplinary clinics even after the COVID-19 pandemic has ended.

\section{References}

1. Ohannessian R, Duong TA, Odone A. Global Telemedicine Implementation and Integration Within Health Systems to Fight the COVID-19 Pandemic: A Call to Action. JMIR Public Health Surveill 2020; 6 (2): e18810. DOI: 10.2196/18810.

2. Stokes EK, Zambrano LD, Anderson KN et al. Coronavirus disease 2019 case surveillance-United States, January 22-May 30, 2020. MMWR Morb Mortal Wkly Rep 69:759, 2020.

3. Coronavirus Disease 2019 (COVID-19) 2020. https://www.cdc.gov/ coronavirus/2019-ncov/index.html.

4. Mitchell EP. Declines in Cancer Screening During COVID-19 Pandemic. J Natl Med Assoc 2020; 112 (6): 563-564. DOI: 10.1016/j.jnma.2020.12.004.

5. Al-Quteimat OM, Amer AM. The Impact of the COVID-19 Pandemic on Cancer Patients. Am J Clin Oncol 2020; 43 (6): 452-455. DOI: 10.1097/ COC.0000000000000712.

6. Rivera A, Ohri N, Thomas $\mathbf{E}$ et al. The impact of COVID-19 on radiation oncology clinics and cancer patients in the US. Adv Radiat Oncol 2020 (5): 538-543. DOI: org10.1016/j.adro.2020.03.006.

7. Nagar H, Formenti SC. Cancer and COVID-19 - potentially deleterious effects of delaying radiotherapy. Nat Rev Clin Oncol 2020; 17 (6): 332-334. DOI: 10.1038/s41571-020-0375-1.

8. Forbes N, Smith ZL, Spitzer RL et al. Changes in Gastroenterology and Endoscopy Practices in Response to the Coronavirus Disease 2019 Pandemic: Results From a North American Survey. Gastroenterology 2020; 159 (2): $772-$ 774.e13. DOI: 1053/j.gastro.2020.04.071.

9. Patt D, Gordan L, Diaz M et al. Impact of COVID-19 on Cancer Care: How the Pandemic Is Delaying Cancer Diagnosis and Treatment for American Seniors. JCO Clin Cancer Inform 2020; 4: 1059-1071. DOI: 10.1200/CCI.20.00134.

10. London JW, Fazio-Eynullayeva E, Palchuk MB, Sankey P, McNair C. Effects of the COVID-19 Pandemic on Cancer-Related Patient Encounters. JCO Clin Cancer Inform 2020; 4, 657-665. DOI: 10.1200/CCI.20.00068.

11. Blue R, Yang A, Zhou C, De Ravin E, Teng C.W, Arguelles GR, Huang V, Wathen C, Miranda SP, Marcotte P, Malhotra NR, Welch WC, Lee JYK. Telemedicine in the Era of Coronavirus Disease 2019 (COVID-19): A Neurosurgical Perspective. World Neurosurg 2020; 139: 549-557. DOI: 10.1016/j.wneu.2020.05.066.

12. Patt DA, Wilfong $\mathbf{L}$, Toth $S$ et al. Telemedicine in Community Cancer Care: How Technology Helps Patients with Cancer Navigate a Pandemic. JCO Oncol Pract 2021; 17 (1): e11-e15. DOI: 10.1200/OP.20.00815.

13. Ohannessian R. Telemedicine: Potential applications in epidemic situations (Télémédecine : applications potentielles en situations épidémiques). European Research in Telemedicine / La Recherche Européenne en Télémédecine 2015; 4 (3): 95-98. DOI: 10.1016/j.eurtel.2015.08.002.

14. McClelland S 3rd, Mitin T, Jaboin JJ, Ciporen JN. RADIANS: A Multidisciplinary Central Nervous System Clinic Model for Radiation Oncology and Neurosurgery Practice. World Neurosurg 2019; 122: 8-10. DOI: 10.1016/j.wneu.2018.10.083.

15. Sun L, Sanjna S, Le A et al. Rates of COVID-19-related Outcomes in Cancer compared to non-Cancer Patients. Preprint. medRxiv 2020; 2020. 08.14. 20174961. DOI: 10.1101/2020.08.14.20174961.

16. Fieux M, Duret S, Bawazeer N, Denoix L, Zaouche S, Tringali S. Telemedicine for ENT: Effect on quality of care during Covid-19 pandemic. Eur Ann Otorhinolaryngol Head Neck Dis 2020; 137 (4): 257-261. DOI: 10.1016/j. anorl.2020.06.014.

17. Paez W, Gheewala R, McClelland S, Jaboin J, Thomas C, Lucke-Wold, B, Ciporen J. Three-Year Experience of a Multidisciplinary Central Nervous System Clinic Model for Radiation Oncology and Neurosurgery (RADIANS) in a Community Hospital Setting. Journal of Neurosurgical Sciences. In press. 18. Wahezi SE, Duarte RA, Yerra S et al. Telemedicine During COVID-19 and Beyond: A Practical Guide and Best Practices Multidisciplinary Approach for the Orthopedic and Neurologic Pain Physical Examination (published correction appears in Pain Physician 2020; 23(6): 647). Pain Physician 2020; 23(4S): S205-S238.

Received March 2, 2021. Accepted March 9, 2021. 